\title{
On Delaunay Oriented Matroids for Convex Distance Functions*
}

\author{
F. Santos \\ Departamento de Matemáticas, Estadística y Computación, Universidad de Cantabria, \\ E-39071, Santander, Spain \\ santos@matsun1.unican.es
}

\begin{abstract}
For any finite point set $S$ in $E^{d}$, an oriented matroid DOM $(S)$ can be defined in terms of how $S$ is partitioned by Euclidean hyperspheres. This oriented matroid is related to the Delaunay triangulation of $S$ and is realizable, because of the lifting property of Delaunay triangulations.

We prove that the same construction of a Delaunay oriented matroid can be performed with respect to any smooth, strictly convex distance function in the plane $E^{2}$ (Theorem 3.5 ). For these distances, the existence of a Delaunay oriented matroid cannot follow from a lifting property, because Delaunay triangulations might be nonregular (Theorem 4.2(i). This is related to the fact that the Delaunay oriented matroid can be nonrealizable (Theorem 4.2(ii)).
\end{abstract}

\section{Introduction}

In this paper we describe a link between the Delaunay triangulation of a finite point set $S$ in the Euclidean $d$-space $E^{d}$ and a certain oriented matroid $\operatorname{DOM}(S)$ of rank $d+2$, which we call the Delaunay oriented matroid of $S$. For the Euclidean distance, $\operatorname{DOM}(S)$ was introduced by Bland and Las Vergnas in [3] (see also pp. 29-32 of [2]). There, only the planar case is considered and the oriented matroid is described in terms of its circuits. We present a description of $\operatorname{DOM}(S)$ in terms of covectors/cocircuits which seems to us simpler. Our main purpose is to generalize Delaunay oriented matroids to the case of Delaunay triangulations computed with respect to the so-called convex distance functions. A brief introduction to oriented matroids is included.

Definition 1.1. Let $S$ be a finite set of points in the Euclidean space $E^{d}$. We define its Delaunay triangulation $\operatorname{Del}(S)$ to be the following polyhedral complex: the convex

\footnotetext{
* This research was partially supported by the Spanish Grant DGICyT PB 92/0498-C02 and the David and Lucile Packard Foundation.
} 
hull conv $(T)$ of a certain subset $T$ of $S$ is a cell in the Delaunay triangulation of $S$ if and only if a Euclidean hypersphere exists passing through every point of $T$ and having every point of $S \backslash T$ in its exterior.

Delaunay triangulations are named after the Russian mathematician B. Delone [8]. They have become a major subject of study in computational geometry and they are geometrically dual to Voronoi diagrams [1], [13]. Some of the cells in the Delaunay triangulation may not be simplices, if more than $d+2$ points lie in a certain hypersphere. We still use the word "triangulation" in this degenerate case. The fact that Delaunay triangulation is a polyhedral complex is a corollary of the lifting property of Delaunay triangulations [4], [9]:

Proposition 1.2 (Lifting Property). Let $S$ be a finite point set in $E^{d}$. Then the Delaunay triangulation of $S$ coincides with the orthogonal projection of the lower envelope of the point set $\bar{S} \subset \mathbf{E}^{d+1}$ obtained lifting $S$ into the paraboloid of equation $x_{d+1}=\sum_{i=1}^{d} x_{i}^{2}$. That is,

$$
\bar{S}:=\left\{\left(x_{1}, \ldots, x_{d}, x_{d+1}\right) \in E^{d+1} \mid\left(x_{1}, \ldots, x_{d}\right) \in S^{d}, x_{d+1}=\sum_{i=1}^{d} x_{i}^{2}\right\} .
$$

The lifting property holds because the intersection of the paraboloid with any nonvertical hyperplane of $E^{d+1}$ projects down to a hypersphere in $E^{d}$. As a consequence, the oriented matroid $\mathcal{M}_{\bar{S}}$ of affine dependencies of $\bar{S}$ describes the partitions of $S$ induced by hyperspheres. For this reason we call $\mathcal{M}_{\bar{S}}$ the Delaunay oriented matroid of $S$ and denote it $\operatorname{DOM}(S)$. The reader not familiar with oriented matroids can see our introduction to them in Section 2, or consult [2]. The above argument indicates that $\operatorname{DOM}(S)$ can be directly defined from $S$ by hypersphere partitions. Our main results are:

- This direct definition of $\operatorname{DOM}(S)$ generalizes to the case where hyperspheres are substituted by the collections of $K$-circles of a smooth, strictly convex distance function $D_{K}$ in the plane (Theorem 3.5).

- The $K$-Delaunay oriented matroids so obtained may be nonrealizable (Theorem 4.2).

- These distance functions do not have, in general, a lifting property (Theorem 4.2).

We also prove that the conditions smooth, strictly convex, and in the plane in Theorem 3.5 are necessary.

Remark 1.3. Our results can be interpreted as incidence theorems on $K$-circles (scaled translations of a given convex body $K$ in the plane). For example, the following theorem holds for Euclidean circles:

Let $p_{1}, \ldots, p_{8}$ be eight distinct points in the plane. If the following quadruples of points are cocircular: $[1,2,3,4],[3,4,5,6],[5,6,7,8],[7,8,1,2],[1,2,5,6]$ then $[3,4,7,8]$ is also cocircular.

Our construction in the proof of Theorem 4.2 is based on a version of this theorem, where two of the circles have infinite radius. Modifying our construction a little, it can be 
proved that this incidence theorem does not hold when Euclidean circles are substituted by the $K$-circles of any symmetric convex distance function, with the only exception of $K$ being an ellipse.

Remark 1.4. A different approach to Delaunay oriented matroids is implicit in [9], although there oriented matroid formalism is replaced by the related concept of a pseudohyperplane arrangement. Let $S$ be a finite point set in $E^{d}$ and let $D$ be any continuous distance function. For each $p \in S$ consider the pseudohyperplane $H_{p}:=\left\{\left(x, x_{d+1}\right) \in\right.$ $\left.E^{d+1} \mid x_{d+1}=D(x, p)\right\}$ in $E^{d+1}$. Let $\mathcal{H}=\left\{H_{p} \mid p \in S\right\}$ be the arrangement of pseudohyperplanes so obtained. In [9] it is shown that the lower envelope of $\mathcal{H}$ projects down to the Voronoi diagram of $S$ with respect to distance $D$.

The relation to our Delaunay oriented matroids is as follows: any cell $C$ in the hyperplane arrangement $\mathcal{H}$ partitions $S$ in three parts, depending on whether $C$ lies below, on, or above the pseudohyperplane $H_{p}$ of a point $p \in S$. This partition of $S$ coincides with the one obtained with a certain $D$-sphere. Thus, a Delaunay oriented matroid exists if the arrangement $\mathcal{H}$ satisfies certain good-intersection conditions [2, Chapter 4]. Since the intersection of pseudohyperplanes of $\mathcal{H}$ is a lifting of the bisector of the corresponding points of $S$, the results in [6] can provide a different proof of our Theorem 3.5. A Delaunay oriented matroid is realizable if the arrangement $\mathcal{H}$ is stretchable, as is the case for the Euclidean distance (see again [9]).

\section{Delaunay Oriented Matroids. The Euclidean Case}

Oriented matroids are abstractly defined as combinatorial objects satisfying the axioms in Definition 2.2 below. Before introducing this formal definition we look at its geometric meaning, from the point of view of affine point configurations. Technically speaking we consider only acyclic oriented matroids.

A signed subset of a set $S$ is a partition $X=\left(X^{+}, X^{0}, X^{-}\right)$of $S$ into three parts, respectively called the positive, zero, and negative part of $X$. The support (noted $\underline{X}$ ) of a signed subset $X=\left(X^{+}, X^{0}, X^{-}\right)$is defined as $\underline{X}=X^{+} \cup X^{-}$. Let $S \subseteq E^{d}$ be a finite set with $n$ points, which affinely $\operatorname{span} E^{d}$. A (signed) covector of the point configuration $S$ is a signed subset $X=\left(X^{+}, X^{0}, X^{-}\right)$of $S$ such that an affine form $h: E^{d} \rightarrow \mathbf{R}$ exists with $h^{-1}\left(\mathbf{R}_{>0}\right) \cap S=X^{+}, h^{-1}\left(\mathbf{R}_{<0}\right) \cap S=X^{-}$, and $h^{-1}(0) \cap S=X^{0}$ (in other words, covectors are partitions of $S$ induced by hyperplanes, together with the empty signed subset $(\emptyset, S, \emptyset)$ ). A covector is called a cocircuit if it has minimal, nonempty support. Equivalently, if $X^{0}$ affinely spans a hyperplane. The chirotope of $S$ is the map $\chi: S^{d+1} \rightarrow\{+, 0,-\}$ defined by

$$
\chi\left(p_{1}, \ldots, p_{d+1}\right)=\operatorname{sign}\left|\begin{array}{cccc}
1 & 1 & \cdots & 1 \\
p_{1} & p_{2} & \cdots & p_{d+1}
\end{array}\right|
$$

Definition 2.1. The oriented matroid $\mathcal{M}_{S}$ of affine dependencies of the point configuration $S$ is defined as the pair $\left(S, \mathcal{V}_{S}\right)$, where $\mathcal{V}_{S}$ is the set of covectors of $S$. 
Definition 2.2. An oriented matroid is a pair $\mathcal{M}=(E, \mathcal{V})$ where $E$ is a finite set and $\mathcal{V}$ is a collection of signed subsets of $E$ called covectors which satisfy the following axioms:

(V0) $(\emptyset, E, \emptyset) \in \mathcal{V}$.

(V1) $\mathcal{V}=-\mathcal{V}$, where $-\left(X^{+}, X^{0}, X^{-}\right):=\left(X^{-}, X^{0}, X^{+}\right)$.

(V2) Composition: for all $X, Y \in \mathcal{V}$, we have $X \circ Y \in \mathcal{V}$, where

$$
X \circ Y:=\left(X^{+} \cup\left(Y^{+} \cap X^{0}\right), X^{0} \cap Y^{0}, X^{-} \cup\left(Y^{-} \cap X^{0}\right)\right) .
$$

(V3) Vector elimination: for all $X, Y \in \mathcal{V}, e \in X^{+} \cap Y^{-}$, there is a $Z \in \mathcal{V}$ such that

$$
\begin{aligned}
& Z^{+} \subseteq\left(X^{+} \cup Y^{+}\right) \backslash e, \\
& Z^{-} \subseteq\left(X^{-} \cup Y^{-}\right) \backslash e
\end{aligned}
$$

and

$$
(\underline{X} \backslash \underline{Y}) \cup(\underline{Y} \backslash \underline{X}) \cup\left(X^{+} \cap Y^{+}\right) \cup\left(X^{-} \cap Y^{-}\right) \subseteq \underline{Z} .
$$

An oriented matroid $\mathcal{M}=(E, \mathcal{V})$ is called acyclic if $(E, \emptyset, \emptyset) \in \mathcal{V}$. It is called polytopal if $(E \backslash\{e\},\{e\}, \emptyset) \in \mathcal{V}$ for all $e \in E$. The rank of an oriented matroid is the maximum number of covectors of which none has support contained in the union of the supports of the others.

An oriented matroid $\mathcal{M}=(\mathcal{V}, E)$ of rank $r$ in $n$ elements is said to be realizable if there is a map $v: E \rightarrow \mathbf{R}^{r}$ such that $\chi\left(e_{1}, \ldots, e_{n}\right)=\operatorname{sign}\left(\operatorname{det}\left(v\left(e_{1}\right), \ldots, v\left(e_{n}\right)\right)\right)$.

The axiomatic definition of oriented matroids can equivalently be given in terms of a set of cocircuits, or a chirotope (or by other means; see Chapter 3 of [2]). Any of them can be translated into the others. For example, the cocircuits are the covectors with minimal nonempty support. Reciprocally, the covectors are exactly those signed subsets which can be recovered from the cocircuits by the composition operations appearing in axiom (V2). The empty signed subset $(\emptyset, S, \emptyset)$ is considered a covector, but not a cocircuit.

The chirotope of an oriented matroid can be read from the collection $\mathcal{C}$ of cocircuits with the following rules. Let $T \subseteq S$ be a subset of cardinality $d$, and $e, e^{\prime} \in S \backslash T$. Then

$$
\begin{gathered}
\chi(T \cup\{e\})=0 \Leftrightarrow \exists X \in \mathcal{C} \text { such that } T \cup\{e\} \subseteq X^{0}, \\
\chi(T \cup\{e\})=\chi\left(T \cup\left\{e^{\prime}\right\}\right) \neq 0 \\
\Leftrightarrow \quad \exists X \in \mathcal{C} \text { such that } T \subseteq X^{0} \text { and }\left\{e, e^{\prime}\right\} \in X^{+} .
\end{gathered}
$$

For an acyclic oriented matroid, realizability is equivalent to the existence of a set $S=\left\{p_{1}, \ldots, p_{n}\right\} \subseteq E^{r-1}$ such that $\mathcal{M}$ is isomorphic to the oriented matroid $\mathcal{M}_{S}$ of affine dependencies of a point configuration $S$, as in Definition 2.1. In this case the realizing vectors are $v\left(e_{i}\right)=\left(1, p_{i}\right)$. An acyclic, realizable oriented matroid is polytopal if and only if the realizing points are in convex position.

Let $C$ be any hypersphere or hyperplane and let $C^{+}$and $C^{-}$be the two connected components of $E^{d} \backslash C$. Then $C$ induces two opposite signed subsets $X=\left(S \cap C^{+}, S \cap\right.$ 
$\left.C, S \cap C^{-}\right)$and $-X=\left(S \cap C^{-}, S \cap C, S \cap C^{+}\right)$of $S$. The signed subsets obtained in this way, together with the empty signed subset, coincide with the covectors of the oriented matroid $\mathcal{M}_{\bar{S}}=\operatorname{DOM}(S)$ of affine dependencies of the lifted point set $\bar{S}$, as defined in the Introduction.

The rank of $\operatorname{DOM}(S)$ equals $d+2$ if and only if $S$ is not contained in a hypersphere or hyperplane. In this case the cocircuits are those covectors $X=\left(X^{+}, X^{0}, X^{+}\right)$for which a unique hypersphere or hyperplane passes through all points in $X^{0}$. The chirotope of $\operatorname{DOM}(S)$ can be obtained as

$$
\chi\left(p_{1}, \ldots, p_{d+2}\right)=\operatorname{sign}\left|\begin{array}{cccc}
1 & 1 & \cdots & 1 \\
p_{1} & p_{2} & \cdots & p_{d+2} \\
p_{1}{ }^{2} & p_{2}{ }^{2} & \cdots & p_{d+2}{ }^{2}
\end{array}\right|
$$

where $p_{k}^{2}:=\sum_{i=1}^{d} x_{k, i}^{2}$ for a point $p_{k}=\left(x_{k .1}, \ldots, x_{k . d}\right)$.

The determinant defining $\chi$ was called the "InCircle" predicate by Guibas and Stolfi [11] (see also Section 17 of [14]) and used as the basic primitive for computing Delaunay triangulations. Its value is zero if and only if the points lie in a hyperplane or hypersphere. If not, let $p_{1}, \ldots, p_{d+1}$ be in general position and let $C$ be the unique hypersphere passing through them. Then $\chi\left(p_{1}, \ldots, p_{d+2}\right)$ is positive if and only if $p_{d+2}$ lies in the "positive" component of $E^{d} \backslash C$ with respect to the orientation defined in $C$ by the ordering of $p_{1}, \ldots, p_{d+1}$. For a circle $C$ passing through the points $p_{1}, p_{2}$, and $p_{3}$ in the plane $E^{2}$, by convention we take the positive component of $E^{2} \backslash C$ to be the one at the left side of $C$ when $C$ is walked so that one encounters the points $p_{1}, p_{2}$, and $p_{3}$ in this order.

A description of $\operatorname{DOM}(S)$ at the level of circuits can be found on pp. 29-32 of [2], for the planar case.

Remark 2.3. Cells in the Delaunay triangulation are convex hulls of subsets $T$ of $S$ such that $(S \backslash T, T, \emptyset)$ is a covector of $\operatorname{DOM}(S)$. Nevertheless, the converse is not true because such a covector can correspond to a hypersphere passing through $T$ and having all $S \backslash T$ inside, instead of outside.

To solve this ambiguity we can extend the covectors in $\operatorname{DOM}(S)$ with an extra point noted $\infty$ and supposed to lie on any hyperplane and outside any hypersphere. The extended covectors still define an oriented matroid, which we call the extended Delaunay oriented matroid of $S$ (and note $\operatorname{EDOM}(S)$ ). A signed subset, $(S \cup\{\infty\} \backslash T, T, \emptyset)$, where $T \subset S$, is a covector of $\operatorname{EDOM}(S)$ if and only if $T$ defines a cell in the Delaunay triangulation of $S$. Moreover, $\operatorname{EDOM}(S)$ contains the combinatorial information of the Voronoi diagram of $S$ of any order $k$ (see [9]). For example, a subset $T \subseteq S$ of $k$ points defines a nonempty cell in the Voronoi diagram of order $k$ of $S$ if and only $(T, \emptyset, S \cup \infty \backslash T)$ is a covector of $\operatorname{EDOM}(S)$. This is related to the fact that the $k$-level of the arrangement of pseudohyperplanes mentioned in Remark 1.4 projects down to the Voronoi diagram of order $k$.

The extended Delaunay oriented matroid is again acyclic and polytopal. It has rank $d+2$ if and only if $S$ affinely spans $E^{d}$. If we delete the point $\infty$ from it we obtain $\operatorname{DOM}(S)$. If we contract $\operatorname{EDOM}(S)$ at the point $\infty$, we obtain the usual oriented matroid $\mathcal{M}_{S}$ of affine dependencies of $S$. 


\section{The Case of Convex Distance Functions}

This section is devoted to introduce convex distance functions and to prove the existence of Delaunay oriented matroids for smooth, strictly convex distance functions in the plane (Theorem 3.5). Also, examples showing the necessity of the three conditions (smooth, strictly convex, and in the plane) are given, as well as a more abstract setting in which Delaunay oriented matroids can be defined (Proposition 3.8).

Definitions 3.1. Let $K$ be a convex body in the Euclidean space $E^{d}$, bounded and with the origin in its interior. The convex distance function $D_{K}(p, q)$ between two arbitrary points $p$ and $q$ is defined to be the unique scaling factor $0<\lambda<\infty$ for which $q$ lies in $p+\lambda \partial K$, where $\partial$ denotes the topological boundary operator. The scaled translations $p+\lambda \partial K$ of $K$ are called $K$-spheres (or $K$-circles if $d=2$ ). In particular, $\partial K$ is called the unit $K$-sphere and $K$ is called the unit $K$-ball.

A convex distance function $D_{K}$ is called strictly convex if $\partial K$ does not contain three collinear points. It is called smooth if $K$ has a unique supporting hyperplane at each boundary point.

Convex distance functions include all the Minkowski metrics (in particular $L_{p}$ distances), which are obtained if $K$ is centrally symmetric. If $K$ is not centrally symmetric, then $D_{K}$ is not a proper metric but it still satisfies the triangle inequality $D_{K}\left(p_{1}, p_{2}\right)+$ $D_{K}\left(p_{2}, p_{3}\right) \geq D_{K}\left(p_{1}, p_{3}\right)$. Convex distance functions in the plane were introduced in computational geometry by Chew and Drysdale in [5], where a divide-and-conquer strategy is used to compute the Voronoi diagrams they produce. Their Voronoi diagrams have also been studied in [6], [7], [12], and [15].

For a strictly convex distance function $D_{K}$ in the plane, we can define the Delaunay triangulation of a point set $S$ with respect to $D_{K}$ substituting Euclidean hyperspheres by $K$-spheres in Definition 1.1. This Delaunay triangulation is a well-defined polyhedral complex. If $D_{K}$ is, in addition, smooth, then any edge in the convex hull of $S$ is a Delaunay edge (see [16]).

To the contrary, for nonstrictly convex distance functions in the plane, or for convex distance functions in dimension $d \geq 3$ whose defining convex body is not an ellipsoid, Definition 1.1 may not yield a polyhedral complex. The main reason for this is that more than one $K$-sphere can pass through $d+1$ points in general position. This gives rise to overlapping cells. The interested reader can easily verify the following example: points $(0,0,2),(0,0,-2),(-1,2,0),(2,-1,0)$ in $E^{3}$ lie in exactly three different $L_{4}$-spheres, with centers along the line $\{z=0, x=y\}$. Even more, this cannot be considered a degeneracy, because it still happens for any small perturbation of the four points. This bad behavior of convex distance functions in 3-space is described in [12] (see also [15]).

Proposition 3.2. Let $D_{K}$ be a convex distance function in $E^{d}, d \geq 2$. For any finite point set $S$, let $\mathcal{V}_{K}(S)$ be the collection of signed subsets of $S$ induced by $K$-spheres (and hyperplanes), together with the empty signed subset. Suppose that $d=2$ and $D_{K}$ is nonsmooth or nonstrictly convex, or that $d>2$ and $K$ is not an ellipsoid. Then there exists a point set $S$ for which $\mathcal{V}_{K}(S)$ is not the set of covectors of an oriented matroid. 
Moreover, $S$ can be found with four points in the nonsmooth case, five in the nonstrictly convex case, and $d+3$ in the case $d>2$.

Proof. The three cases follow from the following general fact: let $p$ and $q$ be two points in $S$. Suppose there exist $K$-spheres $C$ and $D$ with $C \cap S=S \backslash\{p\}$ and $D \cap S=S \backslash\{p, q\}$, but no $K$-sphere contains $S \backslash\{q\}$. Then $\mathcal{V}_{K}(S)$ is not the set of covectors of an oriented matroid. For proof of this, consider the signed subsets coming from $C$ and $D$ with signs given so that $p$ is positive in one and negative in the other: axiom (V3) of Definition 2.2 should produce a covector which is zero in $S \backslash\{q\}$, but not in $q$; this contradicts the hypothesis. We now prove the three cases:

(a) For any nonstrictly convex distance function $D_{K}$ two $K$-circles $C$ and $D$ intersecting in a line segment $\left[p_{1}, p_{2}\right]$ can be found. Let $p_{3}=\left(p_{1}+p_{2}\right) / 2$. Take $q \in C \backslash D$. Finally, take $p \notin\left[p_{1}, p_{2}\right]$ but very close to $p_{3}$ and let $S=$ $\left\{p_{1}, p_{2}, p_{3}, p, q\right\}$.

(b) For a distance $D_{K}$ which is not smooth, three points $p_{1}, p_{2}$, and $p$ which are neither $K$-cocircular nor collinear can always be found. Consider two arbitrary (different) $K$-circles $C$ and $D$ passing through $p_{1}$ and $p_{2}$ and a point $q$ lying in $C$ but not in $D$. Let $S=\left\{p_{1}, p_{2}, p, q\right\}$.

(c) If $d>2$ and $K$ is not an ellipsoid then, according to [10], there exist $d+1$ points $p_{1}, \ldots, p_{d+1}$ not in a hyperplane and which belong to at least two different $K$-spheres $C$ and $D$. Let $p$ be a point in the interior of the simplex $\operatorname{Conv}\left(p_{1}, \ldots, p_{d+1}\right)$. Take $q \in C \backslash D$ and $S=\left\{p_{1}, \ldots, p_{d+1}, p, q\right\}$.

In what follows we are only concerned with smooth, strictly convex distance functions in the plane. It is convenient to compactify the Euclidean space $E^{2}$ into a 2 -sphere $S^{2}$ by adding a point at infinity, noted $\infty$. This additional point lies on every line and in the exterior region of any $K$-circle. In this setting, lines are also considered $K$-circles (they are limit cases).

Our proof of Theorem 3.5 follows from the following properties of $K$-circles.

Lemma 3.3. Let $D_{K}$ be a smooth, strictly convex distance function in the compactified plane $S^{2}=E^{2} \cup\{\infty\}$. Then:

(i) At least one $K$-circle passes through any three points in $S^{2}$.

(ii) Two different $K$-circles intersect in at most two points.

(iii) Let $C$ and $D$ be two $K$-circles which intersect in two points. Then one of the two connected components of $C \backslash D$ lie in each of the two connected components of $S^{2} \backslash D$.

(iv) For any $K$-circle $C$ and any points $p \in C, q \in S^{2}$ the collection $\mathcal{C}_{p, C}$ of $K$-circles intersecting $C$ exactly at $p$ has a unique representative passing through q.

(v) For any point $p$ in $S^{2}$ and any two disjoint $K$-circles $C$ and $D$ not passing through $p$, there is a $K$-circle $E$ passing through $p$ and not intersecting $C$ or D. Moreover, if $p$ is "between" $C$ and $D$ (i.e., in the component of $S^{2} \backslash C$ which contains $D$ and in the component of $S^{2} \backslash D$ which contains $\left.C\right) E$ can be found 
separating $C$ and $D$ (i.e., with $C$ and $D$ contained in different components of $\left.S^{2} \backslash E\right)$.

Proof. Translated to the noncompactified plane, (i) and (ii) are equivalent to "three collinear points are not $K$-cocircular and exactly one $K$-circle passes through any three noncollinear points." For the proof of this see, for example, [12] , [16], or [15]. (The latter gives a more general result in an arbitrary dimension.) Actually, strict convexity is only needed in (ii) and smoothness in (i).

We prove that (iii) actually follows from (i) and (ii). Let $C$ and $D$ be two $K$-circles intersecting at two points $p$ and $q$, so that $C=c_{1} \cup c_{2}$ and $D=d_{1} \cup d_{2}$, where $c_{1}, c_{2}, d_{1}$, and $d_{2}$ are closed arcs joining $p$ to $q$. If (iii) is not true, we can suppose without loss of generality that $c_{1} \cup d_{1}$ separates $c_{2}$ from $d_{2}$. Consider two points $p_{c}$ and $p_{d}$ in $c_{1}$ and $d_{1}$, respectively, and a $K$-circle passing through $p, p_{c}$, and $p_{d}$ (which exists, by (i)). This $K$-circle must intersect either $c_{2}$ or $d_{2}$ in another point, which contradicts (ii).

In (iv) several cases need to be studied. If $p$ is the point at infinity, then $\mathcal{C}_{p, C}$ is the collection of lines parallel to $C$. If $C$ is a line but $p$ is not the point at infinity, then $\mathcal{C}_{p . C}$ contains $C$ and the collection of proper $K$-circles passing through $p$ and having $C$ as supporting line. If $C$ is a proper $K$-circle, then $\mathcal{C}_{p, C}=\mathcal{C}_{p . l}$, where $l$ is the supporting line of $C$ at $p$.

The first part of (v) is trivial, considering a very small $K$-circle passing through $p$ (or a line very far away if $p=\infty$ ). If $p$ is between $C$ and $D$, then the $K$-circle $E$ separating $C$ and $D$ is still easy to find, considering the possible cases for $C$ and $D$ (being proper $K$-circles or lines) and for $p$ (being at infinity or not).

Lemma 3.4. Let $D_{K}$ be a smooth, strictly convex distance function in the compactified plane $S^{2}=E^{2} \cup\{\infty\}$. Let $C$ and $D$ be two $K$-circles (or lines). Denote by $C^{+}$and $C^{-}$ (resp. $D^{+}$and $D^{-}$) the two connected components of $S^{2} \backslash C$ (resp. of $S^{2} \backslash D$ ), and let $p$ be a point in $C^{+} \cap D^{-}$. Then there exists a $K$-circle $E$ such that:

(i) $p \in E$ and $E \cap C=E \cap D=C \cap D$.

(ii) the two connected components of $S^{2} \backslash E$ are contained respectively in $C^{+} \cup D^{+}$ and $C^{-} \cup D^{-}$.

Proof. We consider separately the three possibilities for $C \cap D$ (being two points, one point, or empty).

- If $C$ and $D$ intersect in two points $q$ and $r$, then take $E$ to be the $K$-circle passing through $p, q$, and $r$ (Lemma 3.3(i)). By Lemma 3.3(ii) $E$ satisfies (i). Also, Lemma 3.3(iii) and the fact that $E$ intersects $C^{+} \cap D^{-}$imply that the two components of $E \backslash\{q, r\}$ are contained respectively in $C^{+} \cap D^{-}$and $C^{-} \cap D^{+}$.

- If $C$ and $D$ intersect in one point $q$, then take $E$ to be the unique $K$-circle passing through $p$ in $\mathcal{C}_{q, C}=\mathcal{C}_{q, D}$ (Lemma 3.3(iv)), which trivially satisfies (i). $E \backslash\{q\}$ is connected and does not intersect $C$ or $D$. Since $p \in C^{+} \cap D^{-}$is in $E, E \backslash\{q\}$ must be contained in $C^{+} \cap D^{-}$. This implies that one of the components of $S^{2} \backslash E$ is contained in $C^{+}$and one (maybe the same one) in $D^{-}$. If it is not the same component we have finished. If it is the same one (call it $E_{1}$ and call the other one 
$E_{2}$ ), then we either have $E_{1} \subset C^{+} \subset D^{-}$or $E_{1} \subset D^{-} \subset C^{+}$. This implies that either $C^{-} \cup D^{-}$or $C^{+} \cup D^{+}$equals $S^{2} \backslash\{q\}$, which obviously contains $E_{2}$.

- If $C$ and $D$ do not intersect, then there are essentially two possibilities for the point $p$. If $p$ is between $C$ and $D$, then take as $E$ any $K$-circle passing through $p$ and separating $C$ and $D$. Otherwise, take as $E$ any $K$-circle passing through $p$ and not intersecting $C$ or $D$.

Theorem 3.5. Let $D_{K}$ be a smooth, strictly convex distance function in the compactified plane $S^{2}=E^{2} \cup\{\infty\}$. Let $S$ be a finite point set. Call $\mathcal{V}_{K}(S)$ the collection of signed subsets induced by $K$-circles (and lines), together with the empty signed subset $(\emptyset, S, \emptyset)$. Then $\mathcal{V}_{D_{K}}(S)$ is the set of covectors of an acyclic, polytopal oriented matroid $\mathrm{DOM}_{D_{K}}(S)$. Its rank equals 4 if and only if $S$ is not contained in a $K$-circle (or line). We call this oriented matroid the Delaunay oriented matroid $\operatorname{DOM}_{K}(S)$ of $S$ with respect to distance $D_{K}$.

Proof. Axioms (V0) and (V1) in Definition 2.2 are trivially satisfied for $\mathcal{V}_{K}(S)$. Axioms (V2) and (V3) are also trivial if one of the covectors involved is the empty covector. Thus, consider two covectors $X_{C}$ and $X_{D}$ in $\mathcal{V}_{D_{K}}(S)$ coming from two $D_{K}$-spheres (or lines) $C$ and $D$. Call $C_{+}, C_{-}, D_{+}$, and $D_{-}$respectively the connected components of $S^{2} \backslash C$ and $S^{2} \backslash D$, in the way that agrees with the signs in covectors $X_{C}$ and $X_{D}$. In these conditions the vector elimination axiom (V3) follows directly from Lemma 3.4.

The composition axiom (V2) needs to be proved only in the case that no point of $S$ lies in $U=\left(C_{+} \cap D_{-}\right) \cup\left(C_{-} \cap D_{+}\right)$, according to Corollary 3.7 .7 of [2]. If $U$ is empty, then there is nothing to prove. If $U$ is not empty, then without loss of generality assume that $C_{+} \cap D_{-}$is not empty. Otherwise apply the following to the opposite covectors $-X_{C}$ and $-X_{D}$. Take an additional point $p$ in $C_{+} \cap D_{-}$. Consider the augmented point set $S^{\prime}=S \cup\{p\}$ and the two extended covectors $Y_{C}$ and $Y_{D}$ in $\mathcal{V}_{K}(S)$ induced by the $K$ spheres $C$ and $D$. Since $\{p\}=\left(Y_{C}{ }^{+} \cap Y_{D}{ }^{-}\right) \cup\left(Y_{C}{ }^{+} \cap Y_{D}{ }^{-}\right)$, the covector $Z^{\prime}$ produced by axiom (V3), which we have already proved, is an extension of $X_{C} \circ X_{D}$. Thus, a $K$-circle exists producing $X_{C} \circ X_{D} \in \mathcal{V}_{K}(S)$.

Lemma 3.3(v) implies that a covector $X=\left(X^{+}, X^{0}, X^{-}\right)$with maximal $X^{+}$must equal $(S, \emptyset, \emptyset)$. Thus, $\operatorname{DOM}_{K}(S)$ is acyclic. Lemma 3.3(iv) implies that a covector $X=\left(X^{+}, X^{0}, X^{-}\right)$with $p \in X^{0}$ and maximal $X^{+}$must equal $(S \backslash\{p\},\{p\}, \emptyset)$. Thus, $\operatorname{DOM}_{K}(S)$ is polytopal.

Suppose that there are five nonzero covectors $X_{1}, \ldots, X_{5}$, each with support not contained in the union of the supports of the others. Then there are three points $p_{1} \in \underline{X_{1}}$, $p_{2} \in X_{2}$ and $p_{3} \in X_{3}$ with $p_{1}, p_{2}, p_{3} \notin X_{4} \cup X_{5}$, i.e, the three points lie in the $K$-circles defining $X_{4}$ and $X_{5}$. This implies that $X_{4}$ and $X_{5}$ are equal or opposite, which contradicts the assumption. Thus, the rank of $\operatorname{DOM}_{K}(S)$ is at most 4 . Moreover, if a $K$-circle exists containing $S$, the same argument shows that the rank cannot be 4 , because this would imply the existence of a nonzero covector $X_{4}$ passing through three different points of $S$. If $S$ does not lie in a $K$-circle, take four points in $S$ which are neither collinear nor $K$-cocircular. The four covectors corresponding to the $K$-circles passing through three of them prove that the rank is at least 4 . 
Remark 3.6. The compactified setting makes the extended Delaunay oriented matroid defined in Section 2 be an instance of a Delaunay oriented matroid $\operatorname{DOM}(S)$ in which $S$ contains the point $\infty$. Everything we said in Section 2 for Delaunay oriented matroids (extended or not) in the Euclidean case holds for the Delaunay oriented matroids defined in Theorem 3.5. The only exception is the determinantal formula for computing the chirotope, although the rule for computing it by orienting the $K$-circle passing through three points still holds. A nonempty covector $X$ of $\operatorname{DOM}_{K}(S)$ is a cocircuit if and only if $X^{0}$ has at least three points.

Remark 3.7. Our proof of Theorem 3.5 is completely based on Lemma 3.3. This is emphasized in the following statement which generalizes this fact to arbitrary dimension. The redundant condition (iii) in Lemma 3.3 has been substituted by a new inductive one which applies only to $d>2$. The proof of Proposition 3.8 can be found in [16]. It is similar to our derivation of Theorem 3.5 from Lemma 3.3, with two new technical steps: proving that any nonempty intersection of $\mathcal{D}$-spheres is either a point or a sphere of a certain dimension between 0 and $d-1$ and proving that any $\mathcal{D}$-sphere contains $d+1$ points "in general position", i.e., $d+1$ points not contained in any other $\mathcal{D}$-sphere.

Proposition 3.8. Let $S^{d}$ be a d-dimensional sphere $(d \geq 2)$, and let $\mathcal{D}$ be a collection of $(d-1)$-dimensional spheres (to be called $\mathcal{D}$-spheres) in $S^{d}$. Suppose that $\mathcal{D}$ satisfies the following properties:

(i) At least one $\mathcal{D}$-sphere passes through any $d+1$ points in $S^{d}$.

(ii) Two any nondisjoint $\mathcal{D}$-spheres $C$ and $D$ intersect either in $a(d-2)$-dimensional sphere or in a point.

(iii') If $d>2$, then, for any $\mathcal{D}$-sphere $C$, the collection of $(d-2)$-dimensional spheres $\mathcal{D}_{C}:=\{C \cap D \mid D \in \mathcal{D}, C$ and $D$ intersect in more than one point $\}$, considered as a system of spheres in $C$, again satisfies conditions (i), (ii), (iii'), (iv), and (v).

(iv) For any $\mathcal{D}$-sphere $C$ and any points $p \in C, q \in S^{d}$ the collection $\mathcal{D}_{p, C}$ of $\mathcal{D}$-spheres intersecting $C$ exactly at $p$ has a unique representative passing through $q$.

(v) For any point $p$ in $S^{d}$ and any two disjoint $\mathcal{D}$-spheres $C$ and $D$ not passing through $p$, there is a $\mathcal{D}$-sphere $E$ passing through $p$ and not intersecting $C$ or D. Moreover, if $p$ is "between" $C$ and $D$ (i.e., in the component of $S^{d} \backslash C$ which contains $D$ and in the component of $S^{d} \backslash D$ which contains $\left.C\right) E$ can be found separating $C$ and $D$ (i.e., with $C$ and $D$ contained in different components of $\left.S^{d} \backslash E\right)$.

Let $S \subset S^{d}$ be a finite point set. Call $\mathcal{V}_{\mathcal{D}}(S)$ the collection of signed subsets defined by $\mathcal{D}$-spheres together with the empty signed subset. Then $\mathcal{V}_{K}(S)$ is the set of covectors of an acyclic, polytopal oriented matroid $\operatorname{DOM}_{\mathcal{D}}(S)$. Its rank equals $d+2$ if and only if $S$ is not contained in any $\mathcal{D}$-sphere. 


\section{Nonrealizable Delaunay Oriented Matroids}

In this section we show that the Delaunay oriented matroids appearing in Theorem 3.5 may be nonrealizable. Actually, we see that nonrealizable ones appear for any symmetric, smooth, strictly convex distance function whose unit ball is not an ellipse. The symmetry assumption is due to the use of Lemma 4.1 but the result is possibly true without it. We also see that, for the same class of distance functions, no lifting property is possible: we construct a Delaunay triangulation which is not the projection of the lower envelope of any polyhedron. Triangulations with this negative property are called nonregular [17]. The names nonconvex or noncoherent are used by other authors.

Theorem 4.2 is related to Theorem 3 in [7], which says that Voronoi diagrams and Delaunay triangulations for convex distance functions can have combinatorial types forbidden for the Euclidean distance (this result has been extended to the nonsymmetric case in [16]). Note, however, that the Delaunay triangulation in Fig. 1 has the combinatorial type of a Euclidean Delaunay triangulation.

Lemma 4.1. Let $K$ be a compact, centrally symmetric convex body in the plane $\mathbf{E}^{2}$, which is neither a line segment nor the convex hull of an ellipse. Then there exists an ellipse $E$ with the same center as $K$ and such that $E \backslash K$ has at least four connected components.

Proof. If $K$ is a symmetric quadrilateral, then any ellipse passing through $\cdot$ its four vertices satisfies the lemma. Otherwise, consider any three pairs of opposite points in $\partial K$ in strictly convex position and the unique ellipse $E_{0}$ passing through them. We deal separately with the following cases:

(a) If $\partial K$ does not contain an arc of $E_{0}$, then $E_{0} \backslash \partial K$ has (at least) three pairs of opposite connected components. Thus, either at least two of the three pairs of connected components are exterior to $\partial K$, in which case $E_{0}$ satisfies the conditions of the lemma, or at least two of the three pairs of connected components are interior to $\partial K$. In the latter case, let $E$ be an ellipse exterior to $E_{0}$ but sufficiently close to it and with the same center.

(b) Suppose that $\partial K$ contains an arc of $E_{0}$ and at least one point $x$ exterior to $E_{0}$. Let $p$ be an interior point in the intersection arc. Consider the ellipse $E$ passing through $p$, through $x$, and through their opposite points $p^{\prime}$ and $x^{\prime}$, and having the same tangent as $E_{0}$ at $p$. $E$ satisfies the conditions of the lemma because each of the arcs $p x, x p^{\prime}, p^{\prime} x^{\prime}$, and $x^{\prime} p$ along $E$ have their endpoints in $\partial K$ but contain points exterior to $K$ (namely, those very close to $p$ and $p^{\prime}$ ).

(c) Finally, suppose that $\partial K$ contains an arc of $E_{0}$ and no point exterior to $E_{0}$. Call $p$, $q$, and $r$ three points in such an arc, in this order. Call $s$ a point of $\partial K$ interior to $E_{0}$ (which exists because $\partial K$ is not an ellipse). Then let $E$ be an ellipse passing through $p, q$, and their opposite points $p^{\prime}$ and $q^{\prime}$ and having both $r$ and $s$ (and their opposite points) in its interior.

Theorem 4.2. Let $D_{K}$ be a symmetric, smooth, strictly convex distance function in the plane defined by a convex body $K$. If $K$ is not an ellipse, then there exists a set $S$ of eight 


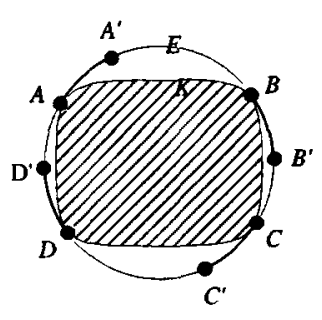

(a)

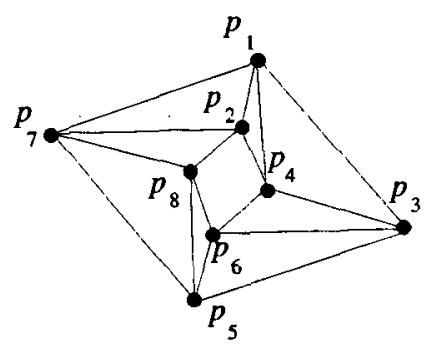

(b)

Fig. 1. Eight points with nonrealizable Delaunay oriented matroids.

points in general position such that:

(i) The K-Delaunay triangulation of $S$ is not the projection of the lower envelope of a polyhedron in three space.

(ii) $\mathrm{DOM}_{K}(S)$ is not realizable.

Proof. We apply Lemma 4.1 to $K$ and, without loss of generality, we suppose that the ellipse $E$ obtained is actually a Euclidean circle (we can make a linear transformation to $K$, if needed). Let $a, b, c$, and $d$ be four different connected components of $E \backslash K, c$ and $d$ being the symmetric copies of $a$ and $b$, respectively. Call $A, B, C$, and $D$ their respective extreme points in the counterclockwise direction. Let $A^{\prime}, B^{\prime}, C^{\prime}$, and $D^{\prime}$ be interior points of $a, b, c$, and $d$, respectively, with segments $\left[A, A^{\prime}\right],\left[B, B^{\prime}\right],\left[C, C^{\prime}\right]$, and $\left[D, D^{\prime}\right]$ being of equal length (see Fig. 1(a)).

Let $S$ consist of the eight points in Fig. 1(b), obtained as

$$
\begin{gathered}
p_{1}=A, \quad p_{2}=D^{\prime}, \quad p_{3}=C^{\prime}, \quad p_{4}=D, \\
p_{5}=p_{4}+\left(D^{\prime}-A^{\prime}\right), \quad p_{6}=p_{4}+\left(A-A^{\prime}\right), \\
p_{7}=p_{6}+\left(A^{\prime}-B^{\prime}\right)=p_{2}+(D-C), \\
p_{8}=p_{6}+\left(B-B^{\prime}\right)=p_{2}+\left(C^{\prime}-C\right) .
\end{gathered}
$$

By construction, points $p_{1} p_{2} p_{5} p_{6}$ and points $p_{3} p_{4} p_{7} p_{8}$ are collinear. Points $p_{1} p_{2} p_{3} p_{4}$, $p_{3} p_{4} p_{5} p_{6}, p_{5} p_{6} p_{7} p_{8}$, and $p_{7} p_{8} p_{1} p_{2}$ are cocircular with respect to Euclidean circles but, with respect to the distance $D_{K}$, their Delaunay triangulation contains all the edges in Fig. 1(b). For example, the unique $K$-circle $C$ passing through points $p_{1}, p_{2}$, and $p_{4}$ must have $p_{3}$ outside because the unit $K$-circle passes through $p_{1}$ and $p_{4}$ and has $p_{2}$ and $p_{3}$ outside. Points $p_{5}, p_{6}, p_{7}$, and $p_{8}$ must also be outside $C$, because of strict convexity. Thus, the three edges $p_{1} p_{2}, p_{2} p_{4}$, and $p_{4} p_{1}$ are Delaunay edges.

We perturb the configuration a little bit, moving the points $p_{1}, p_{3}, p_{5}$, and $p_{7}$ in counterclockwise sense and the points $p_{2}, p_{4}, p_{6}$, and $p_{8}$ in clockwise sense. If the perturbation is sufficiently generic, no three points will be colinear and no four will be $K$-cocircular. For simplicity, we keep the same names for the perturbed points. 
From Fig. 1(b) and the rule for obtaining $\chi[p, q, r, s]$ from the orientation of the $K$-circle passing through $p, q$, and $r$ it is easy to derive the following:

$$
\begin{aligned}
& \chi[1,3,5,2]=\chi[3,5,7,4]=\chi[5,7,1,6]=\chi[7,1,3,8]=-, \\
& \chi[1,3,5,4]=\chi[3,5,7,6]=\chi[5,7,1,8]=\chi[7,1,3,2]=-, \\
& \chi[1,3,5,6]=\chi[3,5,7,8]=\chi[5,7,1,2]=\chi[7,1,3,4]=-,
\end{aligned}
$$

as well as

$$
\begin{aligned}
& \chi[1,2,3,4]=\chi[3,4,5,6]=\chi[5,6,7,8]=\chi[7,8,1,2]=+, \\
& \chi[1,2,6,5]=\chi[3,4,8,7]=+.
\end{aligned}
$$

Note that the two last signs were zero before the perturbation. All the others were nonzero and preserve their sign if the perturbation is sufficiently small.

Suppose that the Delaunay oriented matroid is realizable by eight vectors in $\mathbf{R}^{4}$. Because of general position, we have $\chi[1,3,5,7] \neq 0$. Without loss of generality we assume $\chi[1,3,5,7]=+$ and take the corresponding four vectors as a basis for our coordinate system. Taking into account the signs in (1), the coordinate matrix turns out to be of the form

$$
\left(\begin{array}{cccccccc}
1 & ? & 0 & +a & 0 & +b & 0 & +c \\
0 & -d & 1 & ? & 0 & -e & 0 & -f \\
0 & +g & 0 & +h & 1 & ? & 0 & +i \\
0 & -j & 0 & -k & 0 & -l & 1 & ?
\end{array}\right)
$$

where $a, \ldots, l$ are strictly positive numbers. The signs in (2) imply the inequalities

$$
g k>j h, \quad a l>k b, \quad b f>c e, \quad d i>g f, \quad e j>d l, \quad c h>a i,
$$

which multiplied together give a contradiction. This finishes the proof of (ii).

For (i), suppose that there is a lifting $\left\{q_{1}, \ldots q_{8}\right\} \subset \mathbf{R}^{3}$ of $\left\{p_{1}, \ldots, p_{8}\right\}$ whose lower envelope projects down to our triangulation. Let $l_{1}$ and $l_{2}$ be the lines in the plane passing respectively through the points $p_{1} p_{2} p_{5} p_{6}$ and $p_{3} p_{4} p_{7} p_{8}$ (before the perturbation). Let $v$ be the vertical line through the intersection point of $l_{1}$ and $l_{2}$. We denote by $\pi_{p q r}$ the plane passing through three noncollinear points $p, q$, and $r$ in 3-space. The structure of the triangulation, after perturbation, implies that each of the planes

$$
\pi_{q_{1} q_{2} q_{4}}, \pi_{q_{1} q_{3} q_{4}}, \pi_{q_{3} q_{4} q_{6}}, \pi_{q_{3} q_{5} q_{6}}, \pi_{q_{5} q_{6} q_{\mathrm{s}}}, \pi_{q_{5} q_{7} q_{8}}, \pi_{q_{7} q_{8} q_{2}}, \pi_{q_{7} q_{1} q_{2}}, \pi_{q_{1} q_{2} q_{4}}
$$

intersects the line $v$ in a point below the intersection of the previous one. This gives a contradiction.

\section{Acknowledgments}

This work was conceived while I was visiting the Center for Applied Mathematics at Cornell University in the fall of 1993. For their interest and suggestions I would like to thank Eric Babson, Lou Billera, Jesus de Loera, and Bernd Sturmfels. Also, I would like to thank the referees for many useful comments on the first version of this paper. 


\section{References}

1. F. Aurenhammer, Voronoi diagrams-a survey of a fundamental geometric data structure, ACM Comput. Surv. 23(3) (1991), 345-405.

2. A. Bjorner, M. Las Vergnas, B. Sturmfels, N. White, and G. M. Ziegler, Oriented Matroids, Cambridge University Press, Cambridge, 1992.

3. R. G. Bland and M. Las Vergnas, Orientability of matroids, J. Combin. Theory Ser. B 23 (1978), 94-123.

4. K. Q. Brown, Voronoi diagrams from convex hulls, Inform. Process. Lett. 9 (1979), 223-228.

5. L. P. Chew and R. L. Drysdale III, Voronoi diagrams based on convex distance functions, Proceedings of the 1st ACM Symposium on Computational Geometry, pp. 235-244, 1985.

6. A. G. Corbalán, M. Mazón, and T. Recio, Geometry of bisectors for strictly convex distances, Internat. J. Comput. Geomet. Appl., 6 (1996), 45-58.

7. A. G. Corbalán, M. Mazón, T. Recio, and F. Santos, On the topological shape of planar Voronoi diagrams, Proceedings of the 9 th ACM Symposium on Computational Geometry, pp. 109-115, 1993.

8. B. Delaunay, Sur la Sphère vide, Izv. Akad. Nauk. SSSR VII Ser., Otdel. Mat. Estestv. Nauk 7(6) (1934), 793-800.

9. H. Edelsbruner and R. Seidel, Voronoi diagrams and arrangements, Discrete Comput. Geom., 1 (1986), 25-44.

10. P. R. Goodey, Homothetic Ellipsoids, Math. Proc. Cambridge Philos. Soc., 93 (1983), 25-34.

11. L. Guibas and J. Stolfi, Primitives for the manipulation of general subdivisions and the computation of Voronoi diagrams, ACM Trans. Graphics 4(2) (1985), 74-123.

12. C. Icking, R. Klein, N. M. Le, and L. Ma, Convex distance functions in 3-space are different, Fund. Inform., 22 (1995), 331-352.

13. R. Klein, Concrete and Abstract Voronoi Diagrams, Lecture Notes in Computer Science, Vol. 400, SpringerVerlag, Berlin, 1989.

14. D. E. Knuth, Axioms and Hulls, Lecture Notes in Computer Science, Vol. 606, Springer-Verlag, Berlin, 1992.

15. N. M. Le, On general properties of strictly convex, smooth distance functions in $\mathbf{R}^{D}$, Proceedings of the 5 th Canadian Conference on Computational Geometry, pp. 375-380, 1993.

16. F. Santos, Combinatorial geometry of algebraic curves and Delaunay diagrams in the plane, Ph.D. Thesis (in Spanish), Universidad de Cantabria, 1995.

17. G. M. Ziegler, Lectures on Polytopes, Springer-Verlag, Berlin, 1994.

Received September 25, 1994, and in revised form January 2, 1996. 